Article

\title{
New Promising Targets for Synthetic Omptin-Based Peptide Vaccine against Gram-Negative Pathogens
}

\author{
Valentina A. Feodorova ${ }^{1, *}$, Anna M. Lyapina ${ }^{1}$, Sergey S. Zaitsev ${ }^{1}$, Maria A. Khizhnyakova ${ }^{1}$, \\ Lidiya V. Sayapina ${ }^{2}$, Onega V. Ulianova ${ }^{1}$, Sergey S. Ulyanov ${ }^{3}$ and Vladimir L. Motin ${ }^{4, *}$ \\ 1 Laboratory for Molecular Biology and NanoBiotechnology, Federal Research Center for Virology and \\ Microbiology, Branch in Saratov, 410028 Saratov, Russia; lyapina_anna@inbox.ru (A.M.L.); \\ zaytsev-sergey@inbox.ru (S.S.Z.); khizhnyakova_mariya@mail.ru (M.A.K.); ulianovaov@mail.ru (O.V.U.) \\ 2 Department of Vaccine Control, Scientific Center on Expertise of Medical Application Products, 127051 \\ Moscow, Russia; l.v.sayapina@mail.ru \\ 3 Department for Medical Optics, Saratov State University, 410012 Saratov, Russia; \\ prof.sergey.ulyanov@outlook.com \\ 4 Department of Pathology, Department of Microbiology and Immunology, University of Texas Medical \\ Branch, Galveston, TX 77555, USA \\ * Correspondence: feodorovav@mail.ru (V.A.F.); vlmotin@utmb.edu (V.L.M.)
}

Received: 25 February 2019; Accepted: 4 April 2019; Published: 10 April 2019

check for updates

\begin{abstract}
Omptins represent a family of proteases commonly found in various Gram-negative pathogens. These proteins play an important role in host-pathogen interaction and have been recognized as key virulence factors, highlighting the possibility of developing an omptin-based broad-spectrum vaccine. The prototypical omptin, His-tagged recombinant Pla, was used as a model target antigen. In total, 46 linear and 24 conformational epitopes for the omptin family were predicted by the use of ElliPro service. Among these we selected highly conserved, antigenic, non-allergenic, and immunogenic B-cell epitopes. Five epitopes $(2,6,8,10$, and 11 corresponding to Pla regions 52-60, 146-150, 231-234, 286-295, and 306-311, respectively) could be the first choice for the development of the new generation of target-peptide-based vaccine against plague. The partial residues of omptin epitopes 6, 8, and 10 (regions 136-145, 227-230, and 274-285) could be promising targets for the multi-pathogen vaccine against a group of enterobacterial infections. The comparative analysis and 3D modeling of amino acid sequences of several omptin family proteases, such as Pla (Yersinia pestis), PgtE (Salmonella enterica), SopA (Shigella flexneri), OmpT, and OmpP (Escherichia coli), confirmed their high cross-homology with respect to the identified epitope clusters and possible involvement of individual epitopes in host-pathogen interaction.
\end{abstract}

Keywords: omptin family proteases; Gram-negative pathogens; peptide ELISA; B-cell epitope; peptide vaccine; broad-spectrum vaccine; multi-pathogen vaccine; vaccine development

\section{Introduction}

Currently, infectious diseases (IDs) are responsible for about 30\% of global human mortality per year [1,2]. They are recognized as a significant burden on the public health and economic stability of societies all over the world [3]. During the course of human history, IDs have been accountable for a number of devastating epidemics that have regularly threatened the evolution and even the existence of human civilization [4]. Annually, emerging and neglected IDs affect more than one billion people in more than 149 countries, including chronically infected individuals [5,6]. For instance, diarrheal IDs are the second leading cause of death in children under five years old, and are responsible for killing around 525,000 children every year. Globally, there are nearly 1.7 billion cases of childhood diarrheal IDs every year [7]. 
It is considered that vaccines have achieved extraordinary success in the history of public health, yielding substantial reductions in worldwide morbidity and mortality from IDs over the past half century [8,9]. This has been proven by the accomplishment of smallpox eradication, the drastic reduction in polio cases over the past 20 years, the progress toward tetanus elimination, and the reduction in measles mortality [10]. Vaccination of at-risk populations against plague, tularemia, brucellosis, and a number of other emerging and neglected IDs has been the most effective way to significantly improve the control over disease and deaths from these IDs globally [10,11]. Successful efforts to develop vaccines against acute diarrhea IDs over more than 40 years have resulted in a certain decline in childhood mortality in the past decades [12-14].

Among a number of different types of vaccines, the WHO recognizes four main types, categorized by the antigen used in their preparation: (i) live attenuated (LAV); (ii) inactivated (killed antigen); (iii) subunit (purified antigen); and (iv) toxoid (inactivated toxins). Further, vaccines may be classified as: (i) monovalent, containing a single antigen to protect against a particular ID); (ii) polyvalent, containing two or more variants of the same antigen aiming to protect against a distinct ID; and (iii) combination vaccine, consisting of several individual specific antigens combined in a single injection to prevent the relevant target diseases or to protect against multiple strains of infectious agents causing the same disease [15]. Unfortunately, vaccines based on a single antigen often fail to induce immunity against a group of IDs. In fact, the majority of existing vaccines are carefully targeted at an individual specific antigen substance and are protective only against a single related infectious disease $[10,14,16]$. Moreover, the protective effect of these vaccines is based on duplicating the immune responses induced by natural infections [17]. Recent advances in immunology, structural biology, and synthetic chemistry have heralded the unprecedented rational development of a new generation of effective and safe vaccines that will hopefully be instrumental in controlling many IDs [17-19].

A number of pathogens enter the body via mucosal surfaces using airways, intestines, etc., overcoming innate immunity to establish the infection. This highlights the necessity to induce an efficient mucosal immune response, which is critically important for protection against IDs caused by both foodborne (enteric) and airborne pathogens. Th1 and Th17 cell-mediated immune responses play a critical role in the induction of accelerated and efficient mucosal immune responses. Moreover, the induction of Th17 polarization elicits a protective effect directed to outer membrane proteins (OMPs), providing clade-specific serovar-independent immunity including encapsulated bacteria [20]. Thus, the identification of specific antigens capable of inducing a robust Th17 response to the common prevalent pathogens is one of the critical steps in effective vaccination strategy [21].

Understanding the epitope-antibody interaction is the key for the construction of potent vaccines. B-cell epitope mapping is a promising approach for identifying the main antigenic determinants of microorganisms. Epitope-based vaccines have a remarkable advantage over the conventional ones, since they are safe, strictly specific, able to avoid undesirable immune responses, generate long-lasting immunity, and are reasonably cheaper [22]. In fact, the B-cell epitope mapping of individual target antigen(s) can aid in the identification of both strictly specific and broadly-specific immunodominant epitopes that facilitate the development of both mono-peptide and multi-peptide vaccines against either a single or several IDs. Here we attempted to identify promising molecular targets in the form of linear immunoreactive epitopes suitable for the future development of a new generation of an effective synthetic vaccine against a group of IDs, caused by several Gram-negative pathogens, namely Salmonella, Shigella, Escherichia coli, and Yersinia pestis. These microbes infect both humans and animals and are associated with high levels of morbidity and mortality, an increased emergence of multidrug-resistant strains, as well as with the need to cover protection against different serovars [23].

For this purpose, we investigated the omptins family of outer membrane proteases commonly found in these pathogens that are also known as omptin-expressing bacterial species [24]. These proteins play an important role in host-pathogen interaction and have been recognized as key virulence factors, highlighting the omptins as potential targets for antimicrobial drug and vaccine development [24-26]. The omptin members demonstrate a high level of protein homology (up to 75\%), and have nearly 
identical 3D structures [25]. However, the location of their common immune reactive epitopes has not been elucidated thus far. This study intended to fill in this gap. We used the prototypical omptin as a model target antigen-a His-tagged recombinant Pla (pro-omptin) purified under denaturing conditions [27]. We screened a set of sera from human volunteers who were multiply vaccinated with live plague vaccine (LPV) to identify Pla-specific epitopes [28-30]. To reveal common anti-omptin epitopes, we also used a panel of sera from unvaccinated donors with detectable level of antibodies to Pla who had possible history of prior enteric bacterial diseases. Further, we used a library of 61 overlapping synthetic peptides to map B-cell immune-reactive epitopes to the pro-omptin by peptide ELISA. Finally, we predicted, identified, and proved 11 potential immune-reactive epitopes that are commonly presented in different omptins and could define omptin-mediated host-pathogen interaction. The identification of these commonly-shared immune-reactive epitopes will be helpful in the development of a new generation of effective synthetic omptin-based broad-spectrum peptide vaccine against a number of Gram-negative pathogens.

\section{Material and Methods}

\subsection{Study Design}

A detailed protocol of this study has been published, describing the study design, study population, and methods including consenting procedures, assessment of immune-serological B-cells, and T-cells immune responses of human donors vaccinated with LPV as well as non-vaccinated donors [28-30]. Overall, sera from vaccinated donors $(n=18)$ who received multiple annual immunizations with LPV and unvaccinated healthy individuals $(n=6)$ who had never been in contact with either $Y$. pestis or its antigens participated in the study. Unvaccinated donors including those who had an anamnestic immune response proved their possible previous history of enteric bacterial infections.

The study was conducted in compliance with the Declaration of Helsinki and the regulatory requirements of the Russian Federation. Study protocols were approved by the Institutional Review Board of The Saratov State Medical University named after V. I. Razumovsky (IRB Registration \#: IRB00005197, Federal Wide Assurance (FWA) \#: FWA00009567). Signed written informed consent was obtained from all participants.

\subsection{Omptin Sequences}

The sequences of the omptin proteins, including Pla (Y. pestis CO92), OmpT and OmpP (E. coli), PgtE (Salmonella enterica), and SopA (Shigella flexneri) were downloaded from GenBank (https://www. ncbi.nlm.nih.gov/genbank/). The sequence retrieval accession numbers for each individual omptin along with other information are listed in Table 1.

Table 1. Antigenic and allergenic results of the predicted omptins ${ }^{1}$.

\begin{tabular}{|c|c|c|c|c|c|c|c|}
\hline \multirow[t]{2}{*}{ No. } & \multirow{2}{*}{$\begin{array}{c}\text { GenBank } \\
\text { No. }\end{array}$} & \multirow{2}{*}{$\begin{array}{l}\text { Protein } \\
\text { Name }\end{array}$} & \multirow{2}{*}{$\begin{array}{l}\text { No. of } \\
\text { Amino } \\
\text { Acids }\end{array}$} & \multicolumn{2}{|c|}{$\begin{array}{c}\text { No. of B-Cell Epitopes } \\
\text { Predicted }^{2}\end{array}$} & \multirow[t]{2}{*}{ Allergenicity } & \multirow[t]{2}{*}{ Antigenicity } \\
\hline & & & & Linear & Conformational & & \\
\hline 1 & CAB53170.1 & Pla & 312 & 9 & 6 & Non-allergen & Antigenic \\
\hline 2 & AP001918.1 & OmpP & 315 & 11 & 4 & Non-allergen & Antigenic \\
\hline 3 & KU664810.1 & OmpT & 317 & 7 & 5 & Non-allergen & Antigenic \\
\hline 4 & ATT45876.1 & PgtE & 312 & 10 & 5 & Non-allergen & Antigenic \\
\hline 5 & U73461.1 & SopA & 315 & 9 & 4 & Non-allergen & Antigenic \\
\hline
\end{tabular}




\subsection{Prediction of Linear and Conformational B-Cell Epitopes}

B-cell epitopes were predicted using ElliPro (http://tools.immuneepitope.org/toolsElliPro/) using both protein sequences and structural information for each omptin.

Ribbon diagram models of the three-dimensional (3D) structures of the omptin protein family were generated with Swiss-PdbViewer/DeepView v. 4.1 (https://spdbv.vital-it.ch/) and rendered in POV-Ray v. 3.7. (http://www.povray.org/), based on alignments of the amino acid sequences of Pla, OmpP, OmpT, PgtE, and SopA.

Ribbon diagram models of the 3D structures of the individual predicted immune-reactive omptin protein linear and conformational epitopes were generated by the ElliPro service.

\subsection{Peptide Library Characteristics}

A library of 61 overlapping synthetic peptides, each 15 amino acid residues in length (offset by 5 residues at a time) was made by GenScript, Piscataway, NJ, USA as described recently [30].

\subsection{Predicted Antigenic and Allergenic Characteristics of the Omptin's B-cell Epitopes}

AlgPred [31] (http://crdd.osdd.net), AllerTOP v. 2.0 (http://www.ddg-pharmfac.net/AllerTOP), and ANTIGENpro [32] (http://scratch.proteomics.ics.uci.edu/) services were used for prediction of allergenicity (AlgPred and AllerTop) and antigenicity (ANTIGEN).

\section{Results}

\subsection{Omptin B-Cell Epitopes Prediction}

Overall, ElliPro predicted 46 linear and 24 conformational epitopes for the omptin family (Table 1). The subsequent comparative analysis of the sequences of the pro-omptin Pla with other omptin family proteases, such as PgtE (Salmonella enterica), SopA (Shigella flexneri), and OmpT and OmpP (E. coli) revealed the location of predicted linear B-cell epitopes in either identical positions or in a very close proximity to all nine Pla epitopes predicted by ElliPro and identified serologically using human anti-Pla antisera [30]. Linear epitopes were numbered sequentially from 1 to 11 through the selected proteins of the omptin family. There were at least six linear epitopes common for all five omptins, namely epitopes 1, 2, 5, 7, 8, and 10: four (linear epitopes 4 and 6) for four omptins (omitted in only OmpT); three (linear epitopes 3 and 11) in omptins OmpP, OmpT, and SopA; and epitope 9 in only OmpP and PgtE (Figure 1). The omptin conformational epitopes were formed by the residues of all the relevant predicted linear epitopes for each of the omptins. Additionally, conformational epitopes may include some peptides located at the positions corresponding to one of the 11 omptin linear epitopes that were not predicted for the individual omptin protein. For instance, epitopes 3 and 9 were absent in Pla and were predictable for other omptins, such as OmpP, OmpT, and SopA (epitope 3) and OmpP and PgtE (epitope 9). 


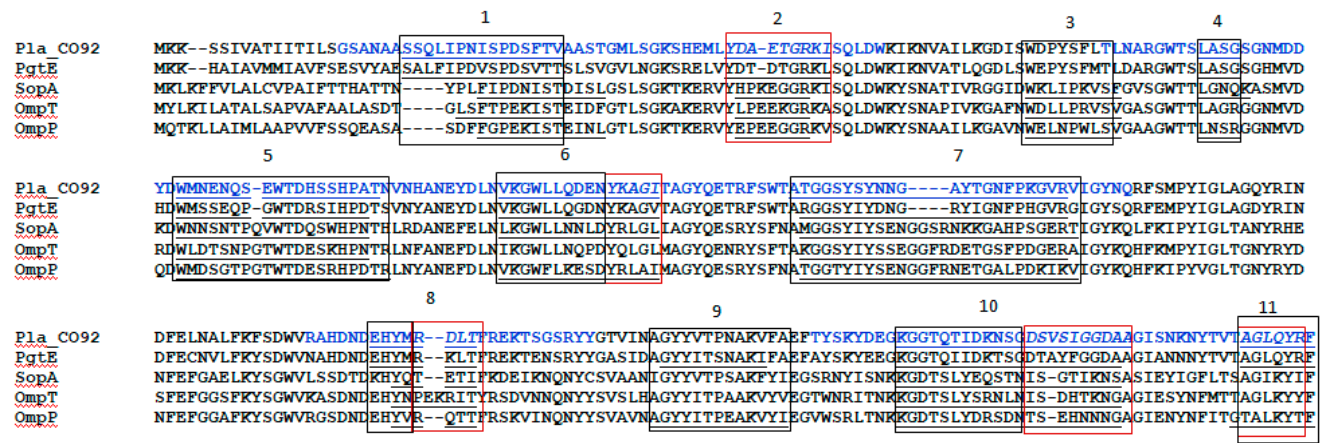

Figure 1. Identification of four major reactive epitopes (indicated in blue) within the pro-omptin molecule and their homology with other omptin family proteases. Alignment of the omptins of different organisms: Pla-Yersinia pestis, PgtE-Salmonella enterica, SopA—Shigella flexneri, OmpT and OmpP-Escherichia coli. Boxes indicate positions of immunodominant regions mapped via immunoreactive peptides.

\subsection{B-Cell Epitope Mapping with Human Sera}

The predicted immuno-reactivity potency of the B-cell linear epitopes were tested and validated by their mapping with the use of a panel of human sera positive in ELISA and immunoblotting for the presence of antibodies to Pla at a detectable level [28-30]. For this purpose a library of 61 overlapping synthetic peptides designed on the basis of the amino acid sequence of the Pla pro-omptin was probed. The initial screening of the peptide library revealed a specific positive reaction of the representative sera samples with 35 out of 61 peptides (Table 2).

Table 2. Immunopositive Pla peptides reacted with sera obtained from vaccinated (group A) and unvaccinated (group B) donors (summarized).

\begin{tabular}{|c|c|c|c|c|c|c|}
\hline \multirow{2}{*}{$\begin{array}{c}\text { Peptide } \\
\text { Immono-Reactive } \\
\text { Cluster No. }\end{array}$} & \multirow{2}{*}{$\begin{array}{l}\text { Omptin Epitope } \\
\text { Predicted in } \\
\text { ElliPro }\end{array}$} & \multirow{2}{*}{$\begin{array}{l}\text { Pla Peptide } \\
\text { Number }\end{array}$} & \multicolumn{2}{|c|}{$\begin{array}{l}\text { Positive Reaction with Sera } \\
\text { from Donor Group }\end{array}$} & \multirow{2}{*}{$\begin{array}{l}\text { Actual Pla Peptide } \\
\text { Position (Library } \\
\text { Peptide ID }{ }^{1} \text { ) }\end{array}$} & \multirow{2}{*}{$\begin{array}{c}\text { Pla Peptide Position } \\
\text { for Epitope Predicted } \\
\text { in ElliPro }\end{array}$} \\
\hline & & & $\mathbf{A}$ & B & & \\
\hline \multirow[b]{2}{*}{1} & 1 & $1-4$ & + & + & $16-45(4-7)$ & $22-36$ \\
\hline & 2 & $5-7$ & + & - & $41-65(9-11)$ & $52-60$ \\
\hline \multirow{2}{*}{$\mathrm{N} / \mathrm{A}^{3}$} & $3^{2}$ & N/A & - & - & $76-90(16)$ & None \\
\hline & 4 & $8-10$ & + & + & $86-110(18-20)$ & $95-98$ \\
\hline \multirow{2}{*}{2} & 6 & 19 & \pm & - & $146-160(30)$ & $136-150$ \\
\hline & 7 & $20-24$ & $\overline{+}$ & + & $156-190(32-36)$ & $163-184$ \\
\hline \multirow[b]{2}{*}{3} & & $26-27$ & + & + & $221-240(45-46)$ & \multirow{2}{*}{$227-234$} \\
\hline & 8 & 28 & + & - & $231-245(47)$ & \\
\hline $\mathrm{N} / \mathrm{A}^{3}$ & $9^{2}$ & N/A & - & - & $251-265$ (51) & None \\
\hline 4 & 10 & $29-32$ & + & + & $266-295(54-57)$ & - \\
\hline
\end{tabular}

${ }^{1}$ described previously [30]; ${ }^{2}$ unreactive peptide in Pla; ${ }^{3}$ Not applicable for Pla (N/A).

These immuno-reactive peptides were grouped into four separate clusters of peptides, suggesting the presence of four major reactive regions within the pro-omptin molecule (Figure 1). These immuno-reactive regions included all nine individual linear B-cell epitopes predicted by using the ElliPro for Pla antigen. Each peptide cluster was formed by several overlapping peptides and occupied either a single or multiple (up to 2-3) separate epitopes. In fact, this provided summarized positive reactions with several different human sera into regions longer that a single predicted epitope. This means that cumulative positive reactions combined from several different human sera reacted with the same epitope formed by several overlapping peptides. No positive reaction was seen with the remaining two epitopes, which were not predicted for Pla while they were found in other omptins 
(epitopes 3 and 9). Importantly, there was 100\% identity of the in silico predicted results with data obtained from the peptide ELISA (Tables 1 and 2, Figure 1).

The subsequent comparative analysis of the sequences of these four clusters of the pro-omptin Pla with other omptin family proteases revealed their cross-homology in the range of $26.6 \%-84.0 \%$ (Figure 2). The highest level of homology (56.0\% \%-84.0\%) was observed with the OmpP protease within clusters $1-4$. The cross-homology with the pro-omptin was at the level of $23.4 \%-80.0 \%$.

The epitopes specific for either Pla alone or the remaining four omptins were selected based on the positive reaction with human sera derived from either vaccinated or unvaccinated donors. Thus, in total there were five epitopes and certain regions of the epitopes specific for Pla only (epitopes 2, 6, 8, 10, and 11) and eight epitopes and their particular regions specific for other omptins (epitopes 1, 5-11). In fact, at least three complete epitopes (e.g., 6, 8, 10) were found to be specific for all five omptins.

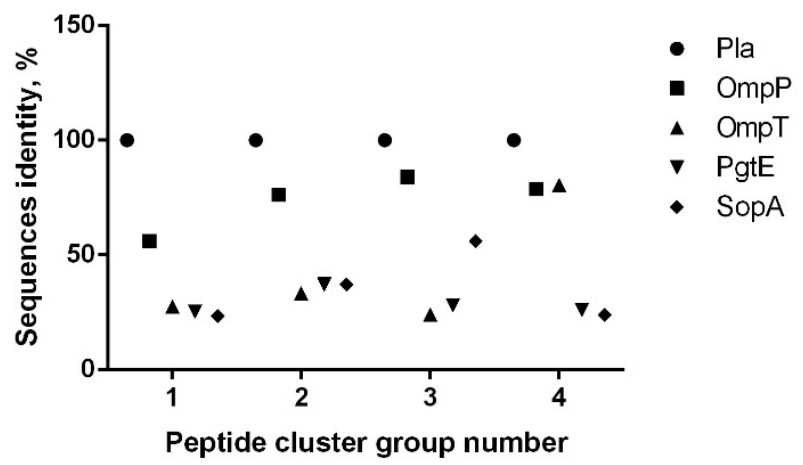

Figure 2. Identification of four major reactive epitopes within the pro-omptin molecule and their homology with other omptin family proteases. Percent homology between amino acid sequences of Pla versus other omptins in four immunodominant regions mapped via immunoreactive peptides.

\subsection{The 3D Modeling of the Predicted Location of the Omptin Epitopes}

We visualized the location of immunoreactive clusters identified during the screening of the omptin peptide sequences ias an the 3D models of different omptins. The comparison of the ribbon diagrams of the 3D models showed high similarity between the omptin structures (Supplementary Materials Figure S1A). All four immuno-reactive regions were positioned within the surface loops. Therefore, the epitopes located in these four clusters could be the best candidates for future immunological and protective studies. The 3D models for individual epitopes of each omptin clearly demonstrated the localization of the linear (Supplementary Materials Figure S1B), conformational (Supplementary Materials Figure S1C) epitopes on the surface loops in Pla and other omptins. These favorable positions of the omptin's epitopes make them available for direct interaction with the relevant homologous specific antibodies present in human sera and probable participation in eliciting host antibody response.

\subsection{Omptins Allergenicity and Antigenicity Prediction}

All five omptins tested were predicted to be non-allergenic while possessing antigenic activity (Table 1). However, there were a few epitopes recognized as probable allergens (Supplementary Materials Table S2), such as epitopes 1 and 3 in OmpT, epitope 9 in OmpP, epitopes 9 and 11 in PgtE, and epitope 3 in SopA. The remaining epitopes were predicted to be non-allergenic. Nevertheless, all the omptin's epitopes were recognized by antisera in ELISA as being antigenic and immuno-reactive.

Finally, all five Pla-specific epitopes were predicted to be non-allergic while the other omptin-specific epitopes 1,9, and 11 could potentially produce allergic reaction against a single omptin epitope (Supplementary Materials Table S2). Thus, the Pla epitope 2 and partial residues of the epitopes 6, 8, 10, and 11 (regions 52-60, 146-150, 231-234, 286-295, and 306-311, respectively) could be the first choice for the development of a new generation of target peptide vaccine against plague. 
The partial residues of omptin epitopes 6, 8, and 10 (regions 136-145, 227-230, and 274-285) could be promising targets for the multi-pathogen vaccine against a group of enterobacterial infections.

\section{Discussion}

IDs caused by omptin-expressing bacterial pathogens remain a significant cause of morbidity and mortality despite the availability of a number of licensed vaccines and many vaccine candidates under different stages of development $[11,16,24,33,34]$. The majority of potential vaccine candidates against enteropathogenic IDs are based on either whole-cell recombinant bacterial strains or different proteins (T3SS, T5SS, outer membrane vesicles, etc.), as well as lipopolysaccharides (LPSs) as potential immunogens. However, the wide serologic diversity of pathogens such as Shigella (four groups of these bacteria are pathogenic in humans, which are further divided into a total of 40 serotypes) [35], Salmonella (the genus includes more than 2400 serovars) [16], and E. coli (pathogenic bacteria are categorized into six pathotypes) [36] significantly limits the development of a universal effective mucosal enteric vaccine. Nevertheless, the main vaccine strategy is focused on the selection of promising candidate(s) with: (i) reduced LPS-associated toxicity, and (ii) ability to confer a broad serovar-independent protection against a group of the relevant IDs [16]. Here, we explored omptins representing a family of outer membrane proteases that have been identified in all major enterobacterial species pathogenic to humans [24] for the presence of similar immuno-reactive epitopes. The model pro-omptin antigen, Y. pestis Pla, was chosen for the following reasons: (i) the antigen is a surface-located outer-membrane integral protease with high similarity in 3D structure to other omptin family members [24]; (ii) Pla can convert plasminogen to plasmin by limited proteolysis, and this activity was likely crucial for the increased lethality of $Y$. pestis during the course of evolution [37-39]; (iii) Pla pro-omptin is involved in the dissemination of $Y$. pestis into circulation and is known as one of the major virulence determinants of this pathogen [40-42]; (iv) this protein has both species-specific and genus-specific epitopes that were detected using a panel of monoclonal antibodies [43]; (v) Pla pro-omptin activation occurs at $37^{\circ} \mathrm{C}$, coinciding with the formation of truncated less-toxic tetra-acylated LPS, which is produced instead of highly toxic hexa-acylated LPS made by $Y$. pestis at $26^{\circ} \mathrm{C}$ [44]. Importantly, the Pla pro-omptin is apparently involved in pathogen-host interaction following the induction of host immune response: a detectable level of the relevant antibodies to this antigen was found in the convalescent sera of human survivors of plague infection [45], in mice that survived experimental plague [46], and in the sera of animals and humans vaccinated with live plague vaccine (LPV, EV line NIIEG) [11,28-30]. Finally, upon vaccination with LPV, there was a detectable level of anti-Pla secretory antibodies of IgA classes [30] suggesting the development of effective mucosal response, which is crucial in developing vaccines for both foodborne and airborne pathogens [21,47]. Importantly, immunization with purified recombinant Pla pro-omptin (i.e., the denatured inactive form of the antigen) provided no protection against plague in a murine model, although partial protection was seen in mice against the strain of $Y$. pestis lacking capsular antigen F1 [48,49]. This could indicate that both linear and conformational epitopes might be involved in eliciting protective effects. The in silico approach used in this study and data obtained by using immuno-serological investigations [30] produced nearly identical results with regard to the identification of immuno-reactive epitopes in omptins. The epitope location fell into four different extended immuno-reactive peptide clusters (Figure 1, Tables 1 and 2) that possessed a high level of homology with other omptins (Figure 2). In fact, using the immuno-bioinformatic tools, we were able to identify several omptin peptides forming highly conserved, antigenic, predicted to be non-allergenic and immunogenic B-cell epitopes, that were least homologous with human host. Further, we narrowed down the extended epitope-containing region to a few immunogenic peptides with a high level of potency to be designed for multi-epitope vaccine candidates against either a group of enteropathogenic IDs or plague only. Moreover, we also found that human immunization with LPV elicited a long-lasting and mixed Th1/Th17 immune responses as determined with pro-omptin as the target antigen [30]. This shift towards Th17 in regularly vaccinated humans was quite possible as Th17 cell-mediated human immunity proved suppressing the IgE responses was associated with human autoimmune 
disease $[50,51]$, because class switch recombination to both $\operatorname{IgG} 4$ and $\operatorname{IgE}$ depends on the production of the Th2 cytokines, particularly IL-4 and should correlate with elevated IL-10 [52]. Also, cytokine profiling revealed a slightly elevated production of IFN- $\gamma$ and diminished level of IL-4 in response of peripheral blood mononuclear cells (PBMCs) to stimulation with Pla [30]. In addition, the sera of immunized donors contained a reliable level of Pla-specific immunoglobulins IgG (IgG1/IgG3) and IgA. All these observations indicate the formation of a typical Th1/Th17 immune response in humans vaccinated with LPV [30], critical for induction of both prompt and long-lasting protective clade specific, as well as serotype-independent mucosal immunity $[20,21]$. This was recently convincingly demonstrated by Chen K. et al. [20] on the model of Klebsiella pneumonia that could be approximated to other omptin-expressing pathogens. Indeed, based on a computer structural 3D model (Supplementary Materials Figure S1A-C) all immune-reactive epitopes detected by us were surface-exposed and could probably be involved in host-pathogen interaction for each omptin-expressing pathogen. This could indicate that some of these immuno-reactive epitopes (predicted or proven) may represent promising targets for the development of a new generation of effective synthetic omptin-based broad-spectrum peptide vaccine against a number of Gram-negative pathogens. Additional studies are necessary to determine individual protective characteristics of each identified epitope after experimental infection with virulent strains of homologous and heterologous enterobacterial pathogens. Moreover, it will be interesting to evaluate the comparative level of IL17A response in patients both actively infected and convalescent after IDs caused by other omptin-expressed pathogens, namely Salmonella, Shigella, and E. coli. On the other hand, the determination of immune correlates of human mucosal immune responses with protection is crucial for the development of new-generation vaccine candidates [47]. One of the challenges in creating universal vaccines is the poor immunogenicity of conserved antigens that was selected during evolution to avoid engaging the immune system. The proposed approach to overcome this deficiency is the structure-based engineering of immunodominant epitopes derived from different variants of the antigen to make a new immunogen capable of eliciting broad protection [53].

\section{Conclusions}

In conclusion, our data suggest excellent progress in the prompt development of a peptide-based vaccine against plague and other enteropathogenic IDs. We also suggest that it could be a single vaccine for multiple pathogens with improved characteristics due to the presence of predicted non-allergenic epitopes. These epitopes could be further evaluated in immunologic and protective studies to assess their potency as promising components of an effective broad-spectrum synthetic mucosal omptin-based peptide vaccine against omptin-expressing pathogens.

Supplementary Materials: The following are available online at http://www.mdpi.com/2076-393X/7/2/36/s1, Figure S1. Structural 3D ribbon diagrams modeling of omptin protein family based on the: (A) original amino acid sequence of Pla, OmpT, OmpP, PgtE, and SopA. The four immune-reactive clusters colored in blue (epitope no. 1), black (epitope no. 2), red (epitope no. 3), and green (epitope no. 4). All models were generated with Swiss-PdbViewer/DeepView v. 4.1. (B) B-cell linear epitopes residues of the Pla, OmpT, OmpP, PgtE, and SopA. (C) B-cell conformational epitopes residues of Pla, OmpT, OmpP, PgtE and SopA. Table S1. Predicted antigenic and allergenic results of the B-cell linear omptins.

Author Contributions: Conceptualization, V.A.F. and V.L.M.; Methodology, V.A.F. and V.L.M.; Analysis, V.A.F., A.M.L., S.S.Z., L.V.S., O.V.U., S.S.U., and V.L.M.; Data Curation, V.A.F., A.M.L., M.A.K., and V.L.M.; Writing-Original Draft Preparation, V.A.F. and V.L.M.; Visualization, S.S.Z.; Supervision, V.A.F. and V.L.M.; Funding Acquisition, V.A.F.

Funding: This work was supported by PFNI RAS, direction 160, project no. 0615-2018-0001.

Acknowledgments: We are grateful to all volunteers who kindly contributed to our study.

Conflicts of Interest: The authors have no conflict of interests to disclose. 


\section{References}

1. World Health Organization. Media Centre. The Top 10 Causes of Death. Available online: http://www.who. int/mediacentre/factsheets/fs310/en/ (accessed on 19 February 2019).

2. Fauci, A.S.; Touchette, N.A.; Folkers, G.K. Emerging infectious diseases: A 10-year perspective from the National Institute of Allergy and Infectious Diseases. Emerg. Infect. Dis. 2005, 11, 519-525. [CrossRef] [PubMed]

3. Nii-Trebi, N.I. Emerging and Neglected Infectious Diseases. Insights, Advances, and Challenges. Biomed. Res. Int. 2017. [CrossRef] [PubMed]

4. Hays, J.N. Epidemics and Pandemics: Their Impacts on Human History; ABC-CLIO: Santa Barbara, CA, USA, 2005; p. 513.

5. Bhutta, Z.A.; Sommerfeld, J.; Lassi, Z.S.; Salam, R.A.; Das, J.K. Global burden, distribution, and interventions for infectious diseases of poverty. Infect. Dis. Poverty 2014, 3, 21. [CrossRef]

6. World Health Organization. Neglected Tropical Diseases. Available online: http://www.who.int/neglected_ diseases/diseases/en/ (accessed on 19 February 2019).

7. World Health Organization. Media Centre. Diarrhoeal Disease. Updated; May 2018. Available online: http://www.who.int/mediacentre/factsheets/fs330/en/ (accessed on 19 February 2019).

8. Plotkin, S.A. Increasing Complexity of Vaccine Development. Infect. Dis. 2015, 212. [CrossRef] [PubMed]

9. Cawein, A.; Emini, E.; Watson, M.; Dailey, J.; Donnelly, J.; Tresnan, D.; Evans, T.; Plotkin, S.; Gruber, W. Human capital gaps in vaccine development: An issue for global vaccine development and global health. Ann. N. Y. Acad. Sci. 2017, 1395, 3-11. [CrossRef] [PubMed]

10. Serdobova, I.; Kieny, M. Assembling a Global Vaccine Development Pipeline for Infectious Diseases in the Developing World. Am. J. Public Health 2006, 96, 1554-1559. [CrossRef]

11. Feodorova, V.A.; Sayapina, L.V.; Corbel, M.J.; Motin, V.L. Russian vaccines against especially dangerous bacterial pathogens. Emerg. Microb. Infect. 2014, 3, e86. [CrossRef]

12. Walker, C.L.; Aryee, M.J.; Boschi-Pinto, C.; Black, R.E. Estimating diarrhea mortality among young children in low and middle income countries. PLoS ONE 2012, 7, e29151. [CrossRef]

13. Walker, C.L.F.; Rudan, I.; Liu, L.; Nair, H.; Theodoratou, E.; Bhutta, Z.A.; O’Brien, K.L.; Campbell, H.; Black, R.E. Global burden of childhood pneumonia and diarrhoea. Lancet 2013, 381, 1405-1416. [CrossRef]

14. O'Ryan, M.; Vidal, R.; del Canto, F.; Salazar, J.C.; Montero, D. Vaccines for viral and bacterial pathogens causing acute gastroenteritis: Part I: Overview, vaccines for enteric viruses and Vibrio cholera. Hum. Vaccin. Immunother. 2015, 11, 584-600. [CrossRef]

15. World Health Organization. Module 2: Types of Vaccine and Adverse Reactions. Available online: http://vaccine-safety-training.org/types-of-vaccine.html (accessed on 19 February 2019).

16. O'Ryan, M.; Vidal, R.; del Canto, F.; Salazar, J.C.; Montero, D. Vaccines for viral and bacterial pathogens causing acute gastroenteritis: Part II: Vaccines for Shigella, Salmonella, enterotoxigenic E. coli (ETEC) enterohemorragic E. coli (EHEC) and Campylobacter jejuni. Hum. Vaccin. Immunother. 2015, 11, 601-619. [CrossRef] [PubMed]

17. Rodrigues, C.M.C.; Pinto, M.V.; Sadarangani, M.; Plotkin, S.A. Whither vaccines? J. Infect. 2017, 74, S2-S9. [CrossRef]

18. Kieny, M.P.; Excler, J.L.; Girard, M. Research and Development of New Vaccines Against Infectious Diseases. Am. J. Public Health 2004, 94, 1931-1935. [CrossRef]

19. Koff, W.C.; Burton, D.R.; Johnson, P.R.; Walker, B.D.; King, C.R.; Nabel, G.J.; Ahmed, R.; Bhan, M.K.; Plotkin, S.A. Accelerating next-generation vaccine development for global disease prevention. Science 2013, 340. [CrossRef] [PubMed]

20. Chen, K.; McAleer, J.P.; Lin, Y.; Paterson, D.L.; Zheng, M.; Alcorn, J.F.; Weaver, C.T.; Kolls, J.K. Th17 cells mediate clade specific, serotype-independent mucosal immunity. Immunity 2011, 35, 997-1009. [CrossRef] [PubMed]

21. Christensen, D.; Mortensen, R.; Rosenkrands, I.; Dietrich, J.; Andersen, P. Vaccine-induced Th17 cells are established as resident memory cells in the lung and promote local IgA responses. Mucosal Immunol. 2017, 10, 260-270. [CrossRef] [PubMed]

22. Ahmada, T.A.; Eweidac, A.E.; Sheweita, S.A. B-cell epitope mapping for the design of vaccines and effective diagnostics. Trials Vaccinol. 2016, 5, 71-83. [CrossRef] 
23. Gayet, R.; Bioley, G.; Rochereau, N.; Paul, S.; Corthésy, B. Vaccination against Salmonella Infection: the Mucosal Way. Microbiol. Mol. Biol. Rev. 2017, 81. [CrossRef] [PubMed]

24. Kukkonen, M.; Korhonen, T.K. The omptin family of enterobacterial surface proteases/adhesins: from housekeeping in Escherichia coli to systemic spread of Yersinia pestis. Int. J. Med. Microbiol. 2004, 294, 7-14. [CrossRef]

25. Brannon, J.R.; Thomassin, J.L.; Gruenheid, S.; Le Moual, H. Antimicrobial Peptide Conformation as a Structural Determinant of Omptin Protease Specificity. J. Bacteriol. 2015, 197, 3583-3591. [CrossRef]

26. Hritonenko, V.; Stathopoulos, C. Omptin proteins: an expanding family of outer membrane proteases in Gram-negative Enterobacteriaceae. Mol. Membr. Biol. 2007, 24, 395-406. [CrossRef]

27. Braciale, V.L.; Nash, M.; Sinha, N.; Zudina, I.V.; Motin, V.L. Correlates of Immunity Elicited By Live Yersinia Pestis Vaccine. In Infectious Disease; Georgiev, V.S., Western, K., McGowan, J.J., Eds.; Springer: Totowa, NJ, USA, 2008; pp. 473-480.

28. Feodorova, V.; Khizhnyakova, M.; Lyapina, A.; Zaitsev, S.; Telepnev, M.; Sayapina, L.; Ulianova, O.; Ulyanov, S.; Arseneva, T.; Morozova, I.; et al. Characterization of Humoral and Cellular Immune Responses to Yersinia Pestis Pla Antigen in Humans Immunised with Live Plague Vaccine (LPV). In Proceedings of the 7th Congress of European Microbiologists (FEMS 2017), Valencia, Spain, 9-13 July 2017.

29. Feodorova, V.A.; Khizhnyakova, M.A.; Lyapina, A.M.; Zaitsev, S.S.; Subbotina, I.A.; Telepnev, M.V.; Sayapina, L.V.; Ulianova, O.V.; Arseneva, T.E.; Morozova, I.V.; et al. New promising targets for synthetic Omptin-based peptide vaccine against Gram-negative pathogens. In Proceedings of the International Society for Vaccine (ISV) Annual Congress, Paris, France, 5-7 October 2017.

30. Feodorova, V.A.; Lyapina, A.M.; Khizhnyakova, M.A.; Zaitsev, S.S.; Sayapina, L.V.; Arseneva, T.E.; Trukhachev, A.L.; Lebedeva, S.A.; Telepnev, M.V.; Ulianova, O.V.; et al. Humoral and Cellular Immune Responses to Yersinia pestis Pla Antigen in Humans Immunized with Live Plague Vaccine. PLoS Negl. Trop. Dis. 2018, 11, e0006511. [CrossRef]

31. Saha, S.; Raghava, G.P. AlgPred: Prediction of allergenic proteins and mapping of IgE epitopes. Nucleic Acids Res. 2006, 34, W202-W209. [CrossRef]

32. Cheng, J.; Randall, A.Z.; Sweredoski, M.J.; Baldi, P. SCRATCH: a protein structure and structural feature prediction server. Nucleic Acids. Res. 2005, 33, W72-W76. [CrossRef]

33. Butler, T. Plague gives surprises in the first decade of the 21st century in the United States and worldwide. Am. J. Trop. Med. Hyg. 2013, 89, 788-793. [CrossRef]

34. Feodorova, V.A.; Motin, V.L. Plague vaccines: current developments and future perspectives. Emerg. Microbes Infect. 2012, 1, e36. [CrossRef]

35. Tulchinsky, T.H.; Varavikova, E.A. Communicable Diseases. In The New Public Health, 3rd ed.; Tulchinsky, T.H., Varavikova, E.A., Eds.; Elsevier/academic Press: San Diego, CA, USA, 2014; pp. 149-236.

36. Center for Disease Control and Prevention. E.coli (Escherichia coli). General Information. Available online: https://www.cdc.gov/ecoli/general/index.html (accessed on 19 February 2019).

37. Haiko, J.; Kukkonen, M.; Ravantti, J.J.; Westerlund-Wikstrom, B.; Korhonen, T.K. The single substitution I259T conserved in the plasminogen activator Pla of pandemic Yersinia pestis branches enhances fibrinolytic activity. J. Bacteriol. 2009, 191, 4758-4766. [CrossRef] [PubMed]

38. Zimbler, D.L.; Schroeder, J.A.; Eddy, J.L.; Lathem, W.W. Early emergence of Yersinia pestis as a severe respiratory pathogen. Nat. Commun. 2015, 6, 7487. [CrossRef] [PubMed]

39. Dentovskaya, S.V.; Platonov, M.E.; Svetoch, T.E.; Kopylov, P.K.; Kombarova, T.I.; Ivanov, S.A.; Shaikhutdinova, R.Z.; Kolombet, L.V.; Chauhan, S.; Ablamunits, V.G. Two Isoforms of Yersinia pestis Plasminogen Activator Pla: Intraspecies Distribution, Intrinsic Disorder Propensity, and Contribution to Virulence. PLoS ONE 2016, 11, e0168089. [CrossRef]

40. Sodeinde, O.A.; Subrahmanyam, Y.V.; Stark, K.; Quan, T.; Bao, Y.; Goguen, J.D. A surface protease and the invasive character of plague. Science 1992, 258, 1004-1007. [CrossRef]

41. Lahteenmaki, K.; Edelman, S.; Korhonen, T.K. Bacterial metastasis: The host plasminogen system in bacterial invasion. Trends Microbiol. 2005, 13, 79-85. [CrossRef]

42. Lathem, W.W.; Price, P.A.; Miller, V.L.; Goldman, W.E. A plasminogen-activating protease specifically controls the development of primary pneumonic plague. Science 2007, 315, 509-513. [CrossRef]

43. Feodorova, V.A.; Devdariani, Z.L. Development, characterisation and diagnostic application of monoclonal antibodies against Yersinia pestis fibrinolysin and coagulase. J. Med. Microbiol. 2000, 49, 261-269. [CrossRef] 
44. Suomalainen, M.; Lobo, L.A.; Brandenburg, K.; Lindner, B.; Virkola, R.; Knirel, Y.A.; Anisimov, A.P.; Holst, O.; Korhonen, T.K. Temperature-induced changes in the lipopolysaccharide of Yersinia pestis affect plasminogen activation by the Pla surface protease. Infect. Immun. 2010, 78, 2644-2652. [CrossRef]

45. Easterbrook, T.J.; Reddin, K.; Robinson, A.; Modi, N. Studies on the immunogenicity of the Pla protein from Yersinia pestis. Contrib. Microbiol. Immunol. 1995, 13, 214-215.

46. Benner, G.E.; Andrews, G.P.; Byrne, W.R.; Strachan, S.D.; Sample, A.K.; Heath, D.G.; Friedlander, A.M. Immune response to Yersinia outer proteins and other Yersinia pestis antigens after experimental plague infection in mice. Infect. Immun. 1999, 67, 1922-1928.

47. Holmgren, J.; Parashar, U.D.; Plotkin, S.; Louis, J.; Ng, S.P.; Desauziers, E.; Picot, V.; Saadatian-Elahi, M. Correlates of protection for enteric vaccines. Vaccine 2017, 35, 3355-3363. [CrossRef]

48. Wang, S.; Heilman, D.; Liu, F.; Giehl, T.; Joshi, S.; Huang, X.; Chou, T.H.; Goguen, J.; Lu, S. A DNA vaccine producing LcrV antigen in oligomers is effective in protecting mice from lethal mucosal challenge of plague. Vaccine 2004, 22, 3348-3357. [CrossRef]

49. Erova, T.E.; Rosenzweig, J.A.; Sha, J.; Suarez, G.; Sierra, J.C.; Kirtley, M.L.; van Lier, C.J.; Telepnev, M.V.; Motin, V.L.; Chopra, A.K. Evaluation of protective potential of Yersinia pestis outer membrane protein antigens as possible candidates for a new-generation recombinant plague vaccine. Clin. Vaccine Immunol. 2013, 20, 227-238. [CrossRef]

50. Noster, R.; Riedel, R.; Mashreghi, M.F.; Radbruch, H.; Harms, L.; Haftmann, C.; Chang, H.D.; Radbruch, A.; Zielinski, C.E. IL-17 and GM-CSF expression are antagonistically regulated by human Thelper cells. Sci. Transl. Med. 2014, 6, 241-280. [CrossRef]

51. Bielinska, A.U.; O'Konek, J.J.; Janczak, K.W.; Baker, J.R., Jr. Immunomodulation of TH2 biased immunity with mucosal administration of nanoemulsion adjuvant. Vaccine 2016, 34, 4017-4024. [CrossRef] [PubMed]

52. James, L.K.; Till, S.J. Potential mechanisms for IgG4 inhibition of immediate hypersensitivity reactions. Curr. Allergy Asthma Rep. 2016, 16. [CrossRef] [PubMed]

53. Rappuoli, R. The challenge of developing universal vaccines. F1000 Med. Rep. 2011, 3, 16. [CrossRef] [PubMed]

(C) 2019 by the authors. Licensee MDPI, Basel, Switzerland. This article is an open access article distributed under the terms and conditions of the Creative Commons Attribution (CC BY) license (http://creativecommons.org/licenses/by/4.0/). 